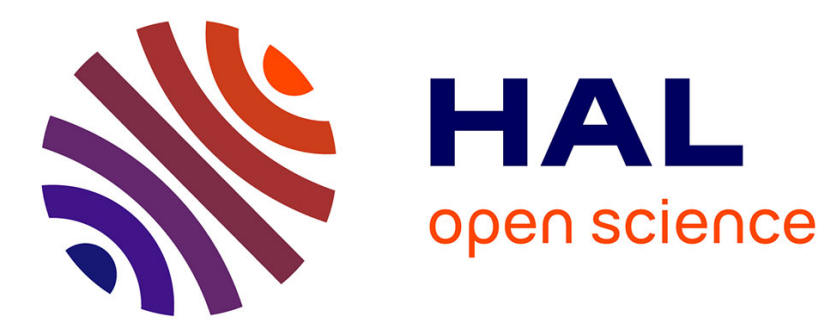

\title{
Filtres par transmission pour l'infrarouge lointain
}

Pierre Quintard, Pierre Delorme

\section{To cite this version:}

Pierre Quintard, Pierre Delorme. Filtres par transmission pour l'infrarouge lointain. Revue de Physique Appliquée, 1967, 2 (4), pp.255-263. 10.1051/rphysap:0196700204025500 . jpa-00242802

\section{HAL Id: jpa-00242802 https://hal.science/jpa-00242802}

Submitted on 1 Jan 1967

HAL is a multi-disciplinary open access archive for the deposit and dissemination of scientific research documents, whether they are published or not. The documents may come from teaching and research institutions in France or abroad, or from public or private research centers.
L'archive ouverte pluridisciplinaire HAL, est destinée au dépôt et à la diffusion de documents scientifiques de niveau recherche, publiés ou non, émanant des établissements d'enseignement et de recherche français ou étrangers, des laboratoires publics ou privés. 


\title{
FILTRES PAR TRANSMISSION POUR L'INFRAROUGE LOINTAIN
}

\author{
Par Pierre QUiNTARD et Pierre Delorme, \\ Faculté des Sciences de Reims, Groupe de Recherches Optiques et Spectroscopiques, \\ Laboratoire de Spectroscopie, B.P. 347, 51-Reims.
}

\begin{abstract}
Résumé. - On décrit deux nouvelles méthodes pour préparer des filtres par transmission, contenant des halogénures alcalins en poudre fine, soit en suspension homogène, soit par strates séparées par des feuilles minces de polyéthylène, et un procédé d'élimination des longueurs d'ondes inférieures à 28 microns. Utilisés sur un spectromètre Ebert-Fastie à petit réseau, desséché par des vapeurs d'azote liquide, ces filtres permettent de couvrir les différentes régions spectrales des premiers ordres de réseaux à 15-8-4 et 2,5 traits $/ \mathrm{mm}$, de 40 à $440 \mu$, et ont déjà permis de nombreuses observations.
\end{abstract}

Abstract. - We describe two new methods for preparing far infrared transmission filters containing alkali halides as a fine powder, either in an homogeneous suspension, or in layers separated by means of thin polyethylene foils, and a process for eliminating unwanted radiations of wavelength below 28 microns. Being used on a compact Ebert-Fastie spectrometer with a small diffraction grating, dried by liquid nitrogen vapour, these filters enable us to cover the different spectral regions of the first orders of gratings with 15-8-4 and $2.5 \mathrm{lines} / \mathrm{mm}$, and have already enabled us to make numerous observations.

Spectromètre. - Le spectromètre utilisé pour ces mesures [1] a déjà permis de nombreuses observations [2], [3], dans un domaine spectral de 40 à $140 \mu$, en utilisant un mode de filtrage décrit par ailleurs [4]. Ge spectromètre (fig. 1) est dérivé dans ses lignes générales de celui réalisé par l'un de nous [5], mais des modifications dans les filtres utilisés nous ont permis d'étendre son domaine d'utilisation jusqu'à $440 \mu$.

Quelques appareils couvrant un domaine spectral s'étendant au-delà de $80 \mathrm{~cm}^{-1}$ ont été décrits, leurs auteurs s'attachant à obtenir soit un pouvoir de résolution élevé [ 6 à 14], soit une résolution voisine de 1 ou de quelques nombres d'ondes [15 à 28], nécessitée par leurs impératifs d'utilisation.

La source est une lampe à vapeur de mercure dont on a montré [29 à 32] qu'elle émettait sensiblement autant que le globar au-delà de $170 \mathrm{~cm}^{-1}$, mais un peu moins aux fréquences plus élevées.

Le monochromateur est de type Ebert-Fastie [33 à 36]; l'étendue géométrique du flux que peut recevoir le détecteur et la résolution optimale que nous nous sommes donnée fixent les dimensions du réseau, et le choix de la distance focale détermine la largeur de la fente [37], [5].

Comme l'a rappelé J. Lecomte [38], et comme nous avons pu le vérifier lors de l'étude de nombreux produits, un pouvoir de résolution de $1 \mathrm{à} 3 \mathrm{~cm}^{-1}$ est suffisant pour l'étude des substances à l'état solide ou liquide. Le détecteur de Golay à fenêtre de quartz peut recevoir des faisceaux d'une ouverture de $60^{\circ}$ sur les $5 \mathrm{~mm}^{2}$ de la partie sensible de la membrane. Une distance focale de $150 \mathrm{~mm}$ permet de réaliser un appareil suffisamment compact, où le trajet des faisceaux lumineux ne sera pas trop grand, tout en simplifiant les réglages optiques. Pour la résolution choisie, de $3 \mathrm{~cm}^{-1}$ à $100 \mathrm{~cm}^{-1}$, on doit alors utiliser avec des réseaux de $68 \times 68 \mathrm{~mm}^{2}$ des fentes de largeur $4,5 \mathrm{~mm}$. L'ensemble optique-détecteur-modulateur est placé dans un caisson étanche.

Le signal issu du détecteur de type Golay [39], [40], [41], amplifié par une unité électronique transistorisée, est reçu sur un enregistreur potentiométrique $(0-10 \mathrm{mV})$. Une constante de temps par résistance et capacité, variable de 5 à $50 \mathrm{~s}$, est adjointe à l'amplificateur et un très bref signal d'une fraction de millivolt, superposé à la tension d'enregistrement, permet de repérer degré par degré la rotation du réseau.

Filtres. - Après la méthode des rayons restants [42], [43], [44], qui a permis la construction des premiers spectromètres pour l'infrarouge lointain, d'autres filtres par réflexion ont été réalisés avec les études de White [45], de Stamm et Whalen [46] sur les réseaux dans l'ordre zéro, puis d'Hadni [47] et Janot [48] sur les réseaux échelettes, de Mitsuishi [49], de Renk, Genzel et Ulrich [50], [51] sur les réseaux grilles, pour lesquels l'étude théorique fut menée par Lewis, Muller, Larsen [52], [53], [54].

Möller [55] a mesuré la transmission de réseaux de polythène et a trouvé que l'effet d'une transmission restait très voisin de l'effet de deux réflexions sur les réseaux échelettes, et Mitsuishi a montré que les réseaux grilles sont de loin les plus intéressants; leur étude expérimentale a été reprise récemment par Möller [10], ils nécessitent cependant la présence d'au moins deux réflexions planes, les réseaux grilles rece- 
vant alors un faisceau de lumière sensiblement parallèle sous faible incidence. Le spectromètre doit donc être conçu en vue de l'utilisation de ces réseaux grilles. Pour que le système de filtrage puisse être adapté à n'importe quel spectromètre, il fallait donc s'orienter vers des filtres par transmission utilisables en faisceau convergent.

Yamada et coll. [56], reprenant une solution préconisée par Ackermann [57], avaient signalé la possibilité de préparer des filtres par transmission, formés par des suspensions d'halogénures, d'oxydes, ou de carbonates de métaux ou de métalloïdes, dans des feuilles de polythène, la fréquence coupée variant avec la substance.

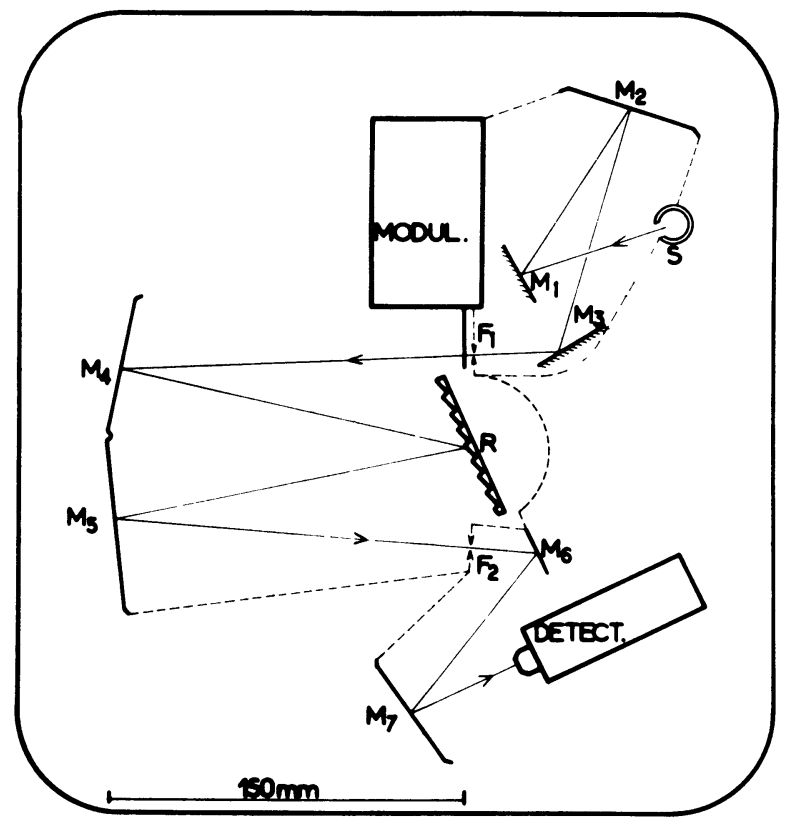

FIG. 1. - Schéma optique du spectromètre.

L'appareillage utilisé par Yamada et coll. a été perfectionné par Russel et Strauss [27], et Möller [58] a récemment indiqué une nouvelle technique ne faisant appel qu'à une presse hydraulique dont le moule peut être porté à une température de $150^{\circ} \mathrm{C}$; l'un d'entre nous [5], puis Lorenzelli [8], a utilisé plus simplement dans le cas du fluorure de sodium une suspension dans de la poudre de polythène pastillée [59], qui donne une courbe de transmission analogue.

Les courbes de transmission ont été données par Yamada et coll. pour différentes quantités de poudre d'oxyde de zinc dans des feuilles de différentes épaisseurs. On note qu'à épaisseur de feuille constante la transmission du filtre augmente lorsque la quantité de $\mathrm{ZnO}$ décroît, mais que, par contre, la région spectrale éliminée décroît, la courbe s'écrasant moins sur l'axe des $\lambda$, alors que, pour un même poids de $\mathrm{ZnO}$, la transmission augmente si les feuilles utilisées sont plus fines.

On pouvait donc prévoir que l'on améliorerait ce procédé de filtrage, s'il était possible soit de diminuer l'épaisseur des feuilles, soit de mieux répartir la poudre utilisée à l'intérieur de la feuille.

Après avoir mis au point une première méthode très simple de fabrication de filtres, qui a donné des résultats comparables à ceux rapportés par Yamada et coll., Möller, Russel et Strauss, c'est vers cette deuxième méthode que nous avons porté notre attention.

L'élimination des longueurs d'ondes inférieures à $28 \mu$ est effectuée ensuite en faisant intervenir une épaisseur minimale de quartz et des pastilles de polythène comprimé d'épaisseur convenable.

Première méthode. - Mode opératoire. - Afin de réaliser des feuilles plus fines que celles obtenues par Yamada et coll., nous avons généralisé la méthode décrite par l'un de nous [5], [59] et dans laquelle la substance, finement broyée, est mélangée, en respectant les pourcentages définis par Yamada et coll., à de la poudre de polythène tamisée. Le mélange est soumis sous vide pendant dix minutes à une pression de $50 \mathrm{kgp} / \mathrm{cm}^{2}$, puis, pendant quelques minutes, à une pression de $120 \mathrm{kgp} / \mathrm{cm}^{2}$. La pastille obtenue, placée entre deux feuilles d'aluminium, qui faciliteront le décollement, est portée, à l'étuve, pendant une demiheure à une température de $150^{\circ} \mathrm{C}$ sous une pression de quelques centaines de grammes $/ \mathrm{cm}^{2}$ entre deux lames planes.

On obtient alors une pastille dont le diamètre est très voisin de celui de la matrice de la presse; si l'on a pris soin de décaler légèrement vers la circonférence de la pastille le centre des forces de pression, les faces de la pastille ne sont plus rigoureusement parallèles et on évite ainsi les franges d'interférence signalées par Möller [58].

RÉsultats. - Les courbes de transmission (fig. 2 et 3) sont voisines de celles données par Yamada pour

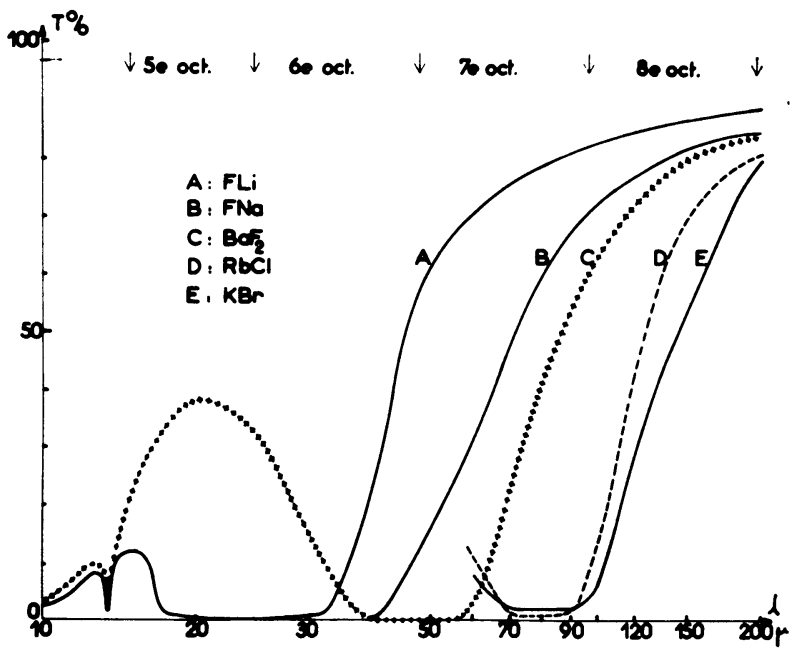

FIG. 2. - Courbes de transmission, méthode I : $\mathrm{FLi}, \mathrm{NaF}, \mathrm{BaF}_{2}: \simeq 4 \mathrm{mg} / \mathrm{cm}^{2}$. $\mathrm{RbCl}, \mathrm{KBr}: \simeq 6,5 \mathrm{mg} / \mathrm{cm}^{2}$. 


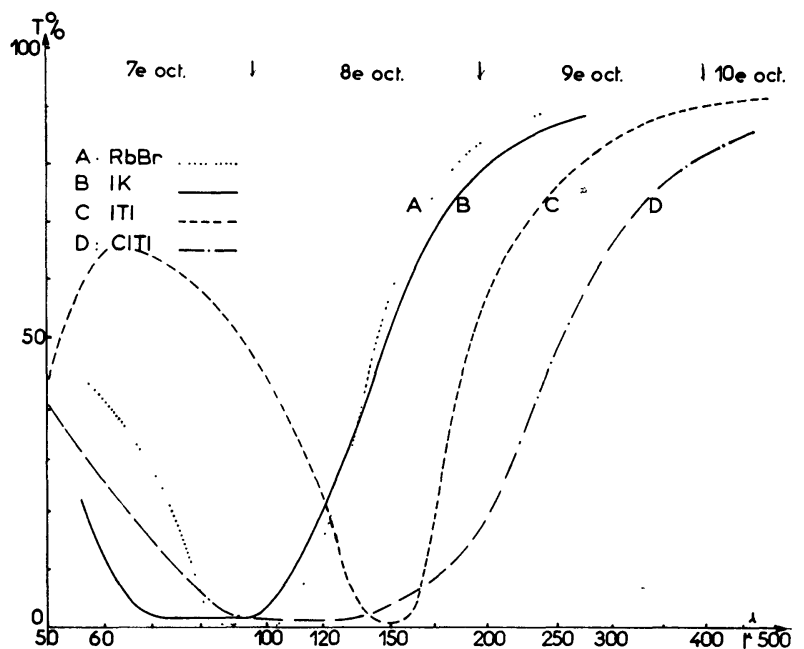

FIG. 3. - Courbes de transmission, méthode I :

$\mathrm{RbBr}, \mathrm{IK}: \simeq 6,5 \mathrm{mg} / \mathrm{cm}^{2}$.

TII, $\mathrm{TlCl}: \simeq 8 \mathrm{mg} / \mathrm{cm}^{2}$.

les produits étudiés par lui, et si l'on a pu dans quelques cas (FLi, FNa...) obtenir des pastilles de 0,20 à $0,25 \mathrm{~mm}$, il paraît, ici aussi, difficile d'obtenir des filtres d'épaisseur inférieure à $0,30 \mathrm{~mm}$ en raison notamment de la fragilité de pastilles plus minces.

Nous avons également représenté les courbes de transmission obtenues par cette méthode avec $\mathrm{ClRb}$ et $\mathrm{BrRb}$.

Deuxième méthode. - Nous avons essayé de réduire, à l'intérieur de la feuille de polythène, l'épaisseur occupée par la poudre cristalline. Pour cela, la poudre très finement broyée est étendue régulièrement sur les deux faces en regard de films de polythène de $30 \mu$ d'épaisseur et l'ensemble est fondu à $160^{\circ} \mathrm{C}$ entre deux feuilles de papier d'aluminium, sous une pression de quelques centaines de grammes. Dans ce cas, les particules sont disposées selon une strate dont l'épaisseur est très voisine du diamètre des grains, $5 \mu$ en moyenne. On a alors la possibilité de réduire notablement le poids de substance disposé par unité de surface; il a pu être ramené au tiers ou au quart de la valeur donnée par Yamada, pour des largeurs de bande coupées identiques.

Nous avons donc repris l'étude des substances citées par Yamada, ainsi que quelques autres qui se sont révélées particulièrement intéressantes, par cette nouvelle méthode, et nous donnons ci-dessous ( fig. 4 à 7 ) les courbes de transmission de quelques filtres que nous avons utilisés sur le spectromètre construit au laboratoire.

FIG. 6. - Courbe de transmission de poudre de [ICs] comprimée en lamelles très fines, maintenue entre deux feuilles de polythène.

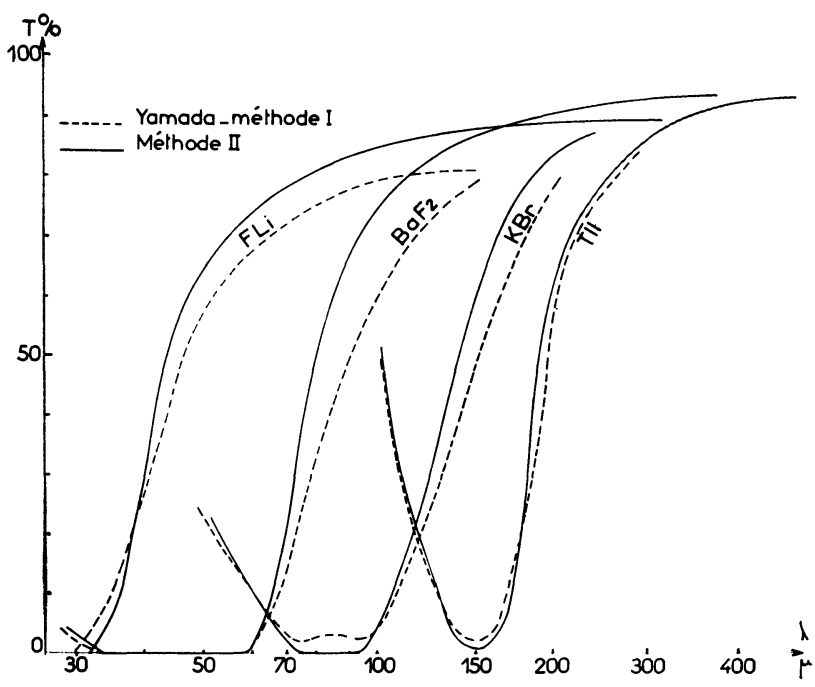

FIG. 4. - Courbes de transmission :

$\mathrm{FLi}: 1 \mathrm{mg} / \mathrm{cm}^{2}, 3 \mathrm{str}$. $-\mathrm{BaF}_{2}: 1,3 \mathrm{mg} / \mathrm{cm}^{2}, 3 \mathrm{str}$. $\mathrm{KBr}: 2 \mathrm{mg} / \mathrm{cm}^{2}, 5 \mathrm{str}$. - TlI : $2 \mathrm{mg} / \mathrm{cm}^{2}, 3 \mathrm{str}$.

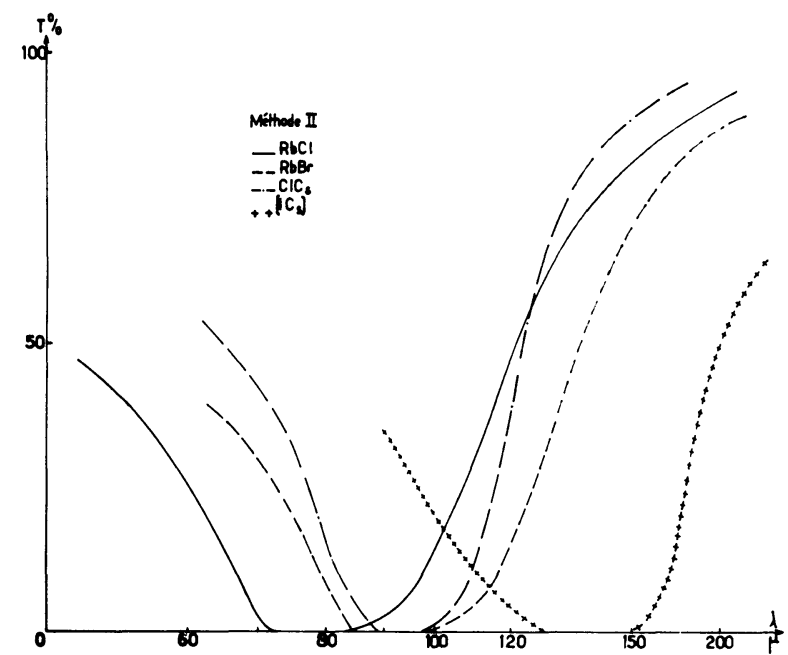

FIG. 5. - Courbes de transmission, méthode II : $\mathrm{RbBr}: 2 \mathrm{mg} / \mathrm{cm}^{2}, 4 \mathrm{str}$. - $\mathrm{RbCl}: 2,5 \mathrm{mg} / \mathrm{cm}^{2}, 4 \mathrm{str}$. $\mathrm{ClCs}: 3 \mathrm{mg} / \mathrm{cm}^{2}, 4 \mathrm{str}$. - [ICs].

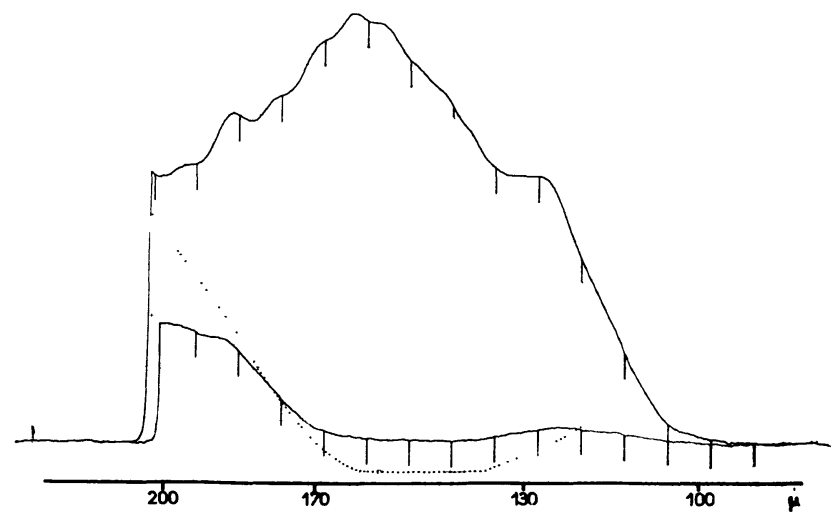

18 


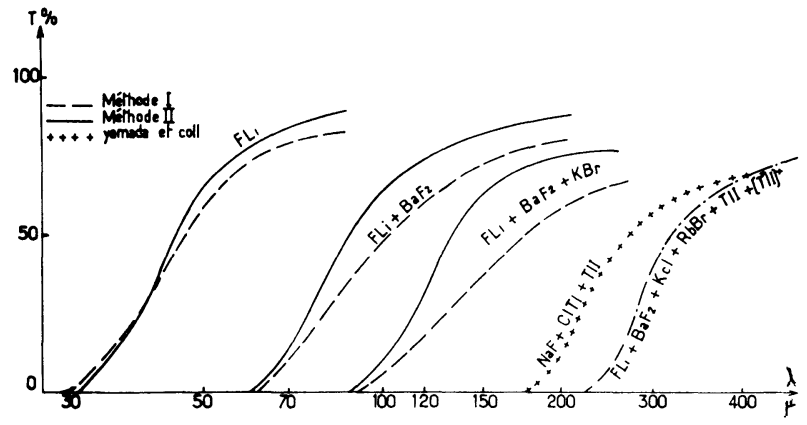

FIG. 7. - Courbes de transmission de quelques combinaisons de filtres utilisés sur le spectromètre.

Mode opératoire. - Pour certaines substances telles que $\mathrm{KBr}, \mathrm{KCl}, \mathrm{ClTl}$, ICs..., la poudre très finement broyée est déposée sur une face du film de polythène et étendue en passant légèrement dessus un doigt ganté lui-même de polythène.

Mais, et c'est surtout le cas des halogénures de métaux lourds, on ne peut dépasser une certaine limite en $\mathrm{mg} / \mathrm{cm}^{2}$ et l'on doit utiliser plusieurs feuilles; leur nombre est précisé pour chaque corps sous la figure correspondante.

Pour d'autres substances telles que FLi, NaF, $\mathrm{BaF}_{2}$, $\mathrm{SrF}_{2}$, on peut en outre utiliser un autre mode opératoire : on découpe dans le film de polythène des cercles du diamètre de la matrice de la presse à pastiller - $25 \mathrm{~mm}-$ et deux pastilles ainsi découpées prennent en sandwich, entre la matrice et le poinçon, une épaisseur de $1 \mathrm{~mm}$ environ de poudre très finement broyée. En réglant la pression, on peut alors incruster dans les deux feuilles une quantité réglable de poudre.

Dans les deux cas, les feuilles sont ensuite mises à l'étuve dans les conditions précisées plus haut dans la première méthode.

Elimination du rayonnement de longueur d'onde inférieure à $\mathbf{4 0}$ microns. - Le procédé le plus couramment employé consiste à utiliser une lame de quartz ou de silice, convenablement noircie, associée à un système diffusant, qui peut être un réseau échelette, un miroir dépoli ou bien, comme l'a rappelé J. Lecomte [60], du polythène dépoli sur ses deux faces.

Dans ce dernier procédé, l'absorption du rayonnement de courte longueur d'onde est due essentiellement au quartz, l'absorption par le polythène ou la diffusion par le dépoli des faces ne jouant qu'un rôle complémentaire pour la région spectrale au-dessous de $2000 \mathrm{~cm}^{-1}$, où le quartz devient à nouveau transparent.

L'absorption du quartz, même sous une épaisseur faible, reste notable surtout dans la septième octave; nous avons donc cherché à réduire au minimum l'épaisseur nécessaire nous limitant aux $0,5 \mathrm{~mm}$ de la fenêtre de la cellule de Golay dont nous disposions; le rayonnement de longueur d'onde inférieure à $5 \mu$, début d'une zone de transmission du quartz, est alors éliminé au moyen d'une pastille de polythène de grain et d'épaisseur convenables. Au-delà de $28 \mu$, le quartz devient à nouveau transparent [43], mais nous disposons des filtres décrits plus haut.

Les pastilles sont préparées selon une méthode déjà décrite [59], et pour déterminer l'épaisseur juste nécessaire pour éliminer le rayonnement parasite, nous enlevons les filtres du spectromètre, à l'exception d'une pastille de $0,5 \mathrm{~mm}$ d'épaisseur, et nous enregistrons la totalité de l'énergie qui, avec un réseau à 8 traits $/ \mathrm{mm}$, tombe sur le détecteur. La même région spectrale est ensuite enregistrée en interposant un écran de $3 \mathrm{~mm}$ de $\mathrm{KBr}$ et $2 \mathrm{~mm}$ de $\mathrm{NaCl}$ dont les fréquences de coupure sont respectivement à $38 \mu$ et $28 \mu$ [43].

On obtient ainsi les courbes de la figure 8 sur lesquelles on note surtout, se dessinant sur un fond

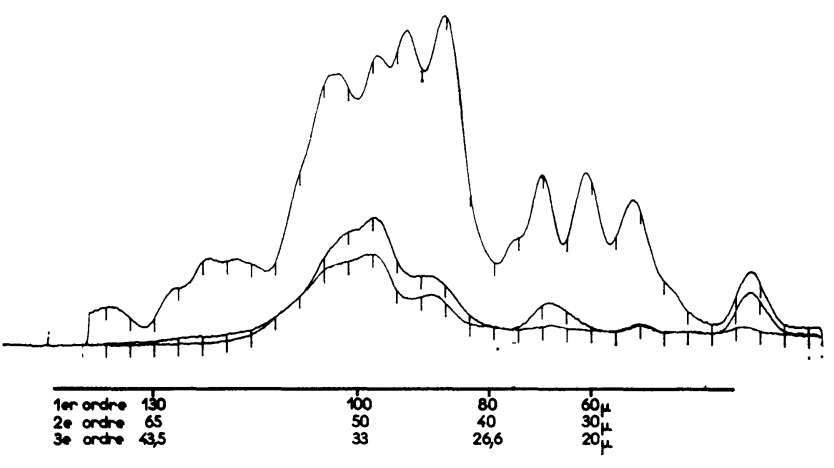

FIG. 8.

Courbe supérieure : énergie totale qui tombe sur le détecteur.

Courbe médiane : énergie parasite transmise par un écran de $3 \mathrm{~mm}$ de $\mathrm{KBr}$.

Courbe inférieure : énergie parasite transmise par un écran de $2 \mathrm{~mm}$ de $\mathrm{NaCl}$.

$$
\begin{gathered}
v_{\text {moy }}=15 \mathrm{~cm}^{-1} / \mathrm{mn} ; \mathrm{RC}=2 \mathrm{~s} ; f=4,5 \mathrm{~mm} \\
\text { Réseau_8_t } / \mathrm{mm} .
\end{gathered}
$$

continu, 3 maximums à $65 \mu, 84 \mu$ et $100 " \mu$ dans le premier ordre ; si le premier peut être attribué essentiellement à $\lambda=33 \mu$ dans le deuxième ordre, les deux autres sont dus à la superposition du troisième ordre et d'ordres supérieurs provenant d'un rayonnement de longueur d'onde inférieur à $5 \mu$.

Nous disposons sur le faisceau, en avant de la cellule de Golay et sur la fente d'entrée du monochromateur, des épaisseurs croissantes de polythène très finement divisé, pastillé; on note alors pour la position $100 \mu$ correspondant au maximum d'énergie parasite une diminution d'intensité du rayonnement étranger à la région spectrale étudiée ( fig. $9 \mathrm{~A}$ ). Avec une épaisseur de $0,75 \mathrm{~mm}$ de polythène devant la fenêtre du détecteur et de $0,60 \mathrm{~mm}$ devant la fente $F_{1}$, le rayonnement 


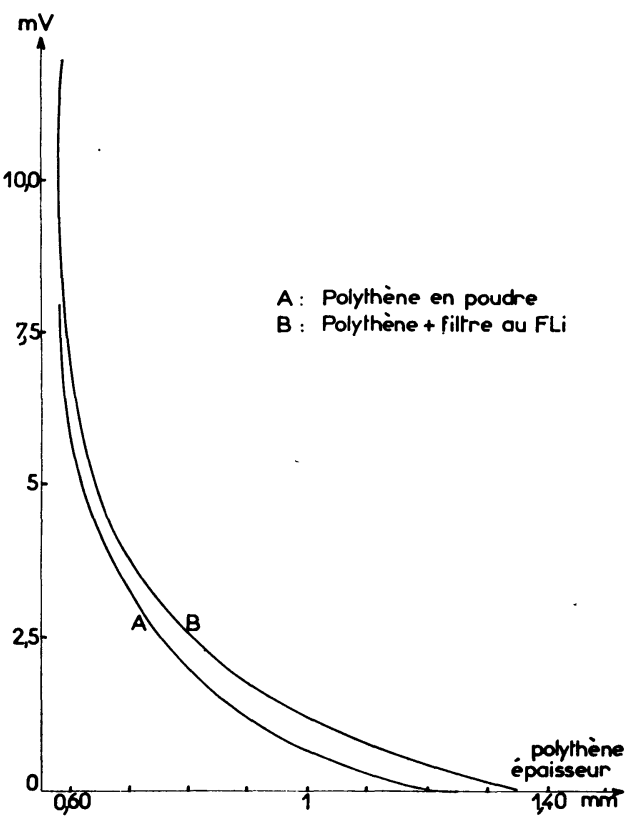

FIG. 9. - Absorption de l'énergie parasite à $100 \mu$.

A) Par des épaisseurs croissantes de polythène.

B) Par FLi + polythène.

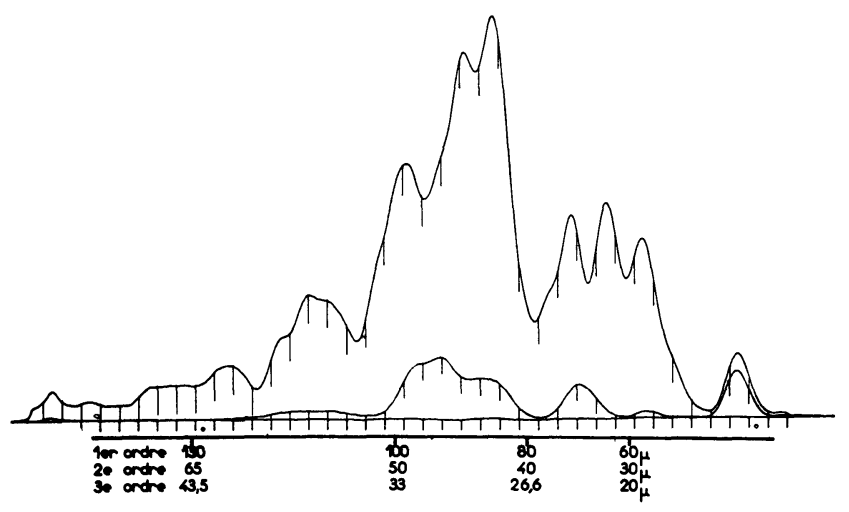

FIG. 10.

Courbe supérieure : énergie totale tombant sur le détecteur.

Courbe inférieure : l'énergie parasite de longueur d'onde $<28 \mu$ se confond avec le bruit de fond.

Courbe médiane : énergie parasite $28 \mu<\lambda<38 \mu$.

$$
v_{\text {moy }}=15 \mathrm{~cm}^{-1} / \mathrm{mn} ; \mathrm{RC}=2 \mathrm{~s} ; f=4,5 \mathrm{~mm}
$$

Réseau $8 \mathrm{t} / \mathrm{mm}$.

de longueur d'onde inférieur à $28 \mu$ se confond avec le bruit de fond (fig. 10).

L'adjonction d'un filtre au fluorure de lithium élimine les longueurs d'ondes jusqu'à $38 \mu$ (fig. 11) et permet de diminuer légèrement l'épaisseur de polythène ( fig. $9 \mathrm{~B})$.

La figure 12 permet d'apprécier le gain très sensible d'énergie que procure ce procédé d'élimination du rayonnement parasite par rapport à celui que nous utilisions précédemment.

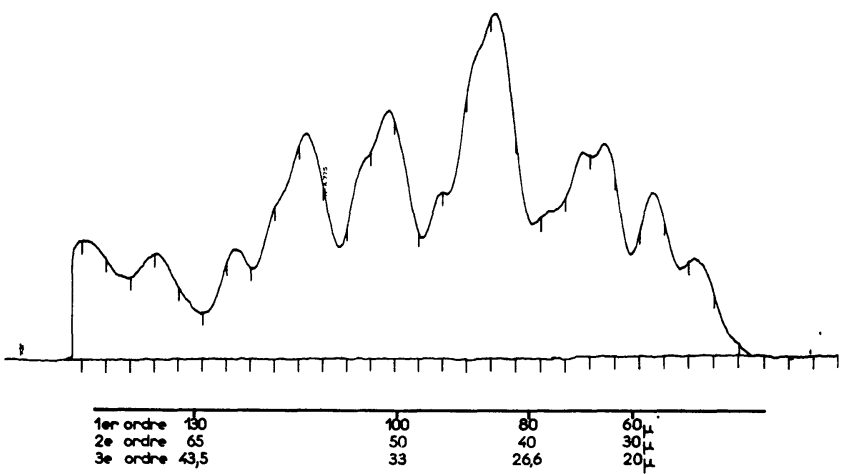

Fig. 11.

Courbe supérieure : énergie totale reçue par le détecteur.

Courbe inférieure : l'énergie parasite de longueur d'onde $<38 \mu$ se confond avec le bruit de fond.

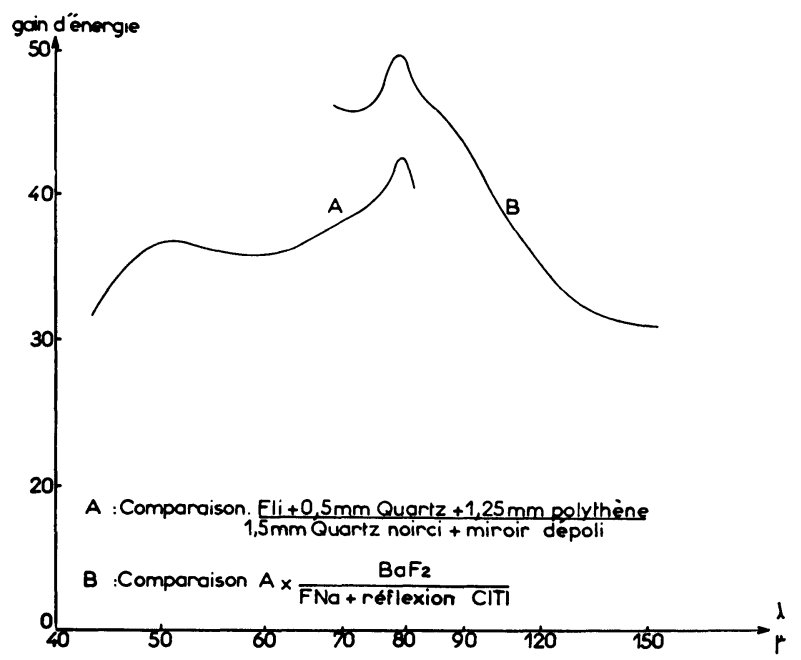

Fig. 12. - Gain d'énergie entre notre méthode et celle citée dans [5] pour la région spectrale commune 45-140 $\mu$.

En résumé, le rayonnement de longueur d'onde inférieur à $38 \mu$ est éliminé au moyen de :

- Filtre par transmission au fluorure de lithium.

- Épaisseur de $0,75 \mathrm{~mm}$ de polythène pastillé devant la fenêtre du détecteur.

- Une épaisseur de $0,50 \mathrm{~mm}$ de polythène pastillé devant la fente $F_{1}$.

Nous donnons (fig. 11) le spectre obtenu avec ce mode de filtrage et l'énergie parasite qui traverse une lamelle de $\mathrm{KBr}$ de $3 \mathrm{~mm}$.

L'énergie parasite est déterminée jusqu'à $80 \mu$ au moyen d'écrans d'halogénures alcalins convenablement choisis [43], [5], et au-delà, nous utilisons une méthode préconisée par Hadni : pour un intervalle spectral donné $I_{1}$ couvert dans le premier ordre d'un réseau à $N$ traits $/ \mathrm{mm}$, l'énergie parasite est recherchée dans les mêmes conditions de gain et de filtres qu'en $I_{1}$ dans le premier ordre d'un réseau à $2 \mathrm{~N}$ traits $/ \mathrm{mm}$. 
Pour la région spectrale, où les mesures comparatives sont possibles $(40-80 \mu)$, nous avons vérifié que cette deuxième méthode était en général aussi sensible que celle des écrans et, au voisinage de la longueur d'onde de coupure des plaquettes d'halogénures, lorsque la transmission décroît fortement, que ce deuxième mode était le seul utilisable, puisqu'il faudrait rechercher dans l'ordre 2 ou 3 la réapparition d'une fraction très faible de l'énergie parasite transmise par la plaquette.

On peut également, sans changer de réseau, noter directement le pourcentage d'énergie parasite trans-

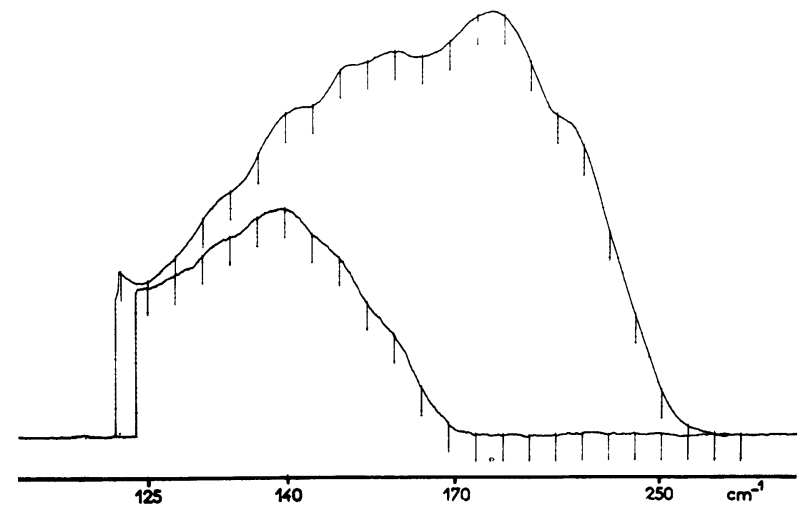

FIG. 13.

Courbe supérieure : FLi.

Courbe inférieure : $\mathrm{FLi}+\mathrm{BaF}_{2}$ (gain modifié).

$$
\mathrm{RC}=10 \mathrm{~s} ; v_{\text {moy }}=8 \mathrm{~cm}^{-1} / \mathrm{mn} ; f=4,5 \mathrm{~mm}
$$

Réseau $15 \mathrm{t} / \mathrm{mm}$.

mise : dans le premier ordre d'un réseau à 15 traits $/ \mathrm{mm}$ dans l'intervalle de longueurs d'ondes (38 à $76 \mu$ ) couvert par un filtre au FLi, l'adjonction d'un filtre au $\mathrm{BaF}_{2}$ élimine toute l'énergie jusqu'à $58 \mu$ (fig. 13).

Toutes ces vérifications n'ont pas permis de déceler d'énergie parasite dont l'intensité soit supérieure au bruit de fond.

Dessèchement. - La partie optique et le détecteur étant contenus dans un caisson parfaitement étanche dans lequel on réalise un vide primaire, il devient alors difficile de faire des mesures à l'état liquide ou en solution : les cuves ordinaires [59] ne résistent pas à une telle pression. Lors d'une étude sur quelques paradisubstitués du benzène [3], nous avions été intrigués par le fait que pour certains de ces corps nous trouvions vers les basses fréquences des bandes d'absorption que les auteurs ne citaient pas [62], [63], [64]; il était alors possible, les produits étudiés étant volatils et les appareils travaillant sous vide, que les corps pastillés se soient sublimés.

De plus, les échantillons sont introduits par un puits centré sur $\mathrm{F}_{2}$; pour résister au vide, il serait nécessaire de sceller des fenêtres d'environ $1 \mathrm{~mm}$ d'épaisseur, donc assez absorbantes.

Nous avons donc envisagé d'éliminer la vapeur d'eau, soit au moyen d'une circulation d'air soigneusement desséché [5], [8], [65] à [69], soit en remplaçant l'air atmosphérique par un gaz inerte; ce procédé mis au point par O. H. Gaebler [70], rappelé par J. Lecomte, a été employé par l'un de nous [5] pour la mesure des bandes de $\mathrm{NH}_{3}$ dilué dans de l'azote $\mathrm{R}$. Nous avons préféré utiliser le gaz, libéré par l'azote liquide contenu dans un dewar dans lequel plonge une résistance; le courant d'azote peut alors varier de 30 à 300 litres/heure. Si le courant maximum doit être utilisé pour accélérer le dessèchement, après que l'on ait ouvert le capot du spectromètre, le débit minimum suffit ensuite à maintenir un dessèchement parfait; 15 litres d'azote liquide sont alors suffisants par 24 heures.

Un spectre obtenu avec et sans dessèchement est donné figure 14; dans ces conditions, les échantillons et les filtres peuvent alors être introduits de l'extérieur par le puits isolé par deux fenêtres de polythène d'épaisseur $30 \mu$; le parcours optique dans l'air n'est que de $1,8 \mathrm{~cm}$ et nous pouvons utiliser les cuves en

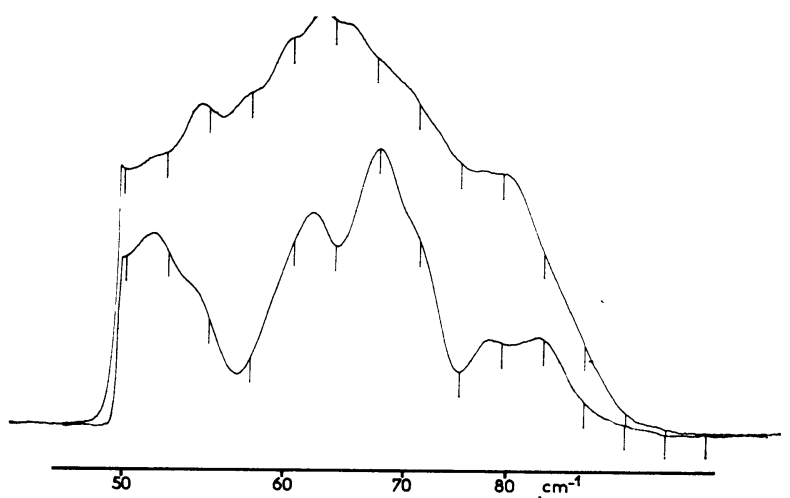

FIG. 14.

Courbe supérieure : sous courant d'azote gazeux. Courbe inférieure : air atmosphérique.

$$
\text { Réseau : } 4 \mathrm{t} / \mathrm{mm}
$$

$$
f=4,5 \mathrm{~mm} ; \mathrm{RC}=20 \mathrm{~s} ; 98 \mu<\lambda<196 \mu \text {. }
$$

polythène déjà citées pour l'étude des liquides et solutions.

La différence entre le dessèchement du caisson par un vide secondaire ou par un courant d'azote est alors de quelques pour cent à notre désavantage, mais nous avons gagné une grande facilité d'emploi et éliminé les inconvénients signalés au début de ce paragraphe.

Les figures 15 à 19 permettent de juger de la résolution obtenue avec ce spectromètre; on notera que le gain très sensible d'énergie que procure ce procédé de filtrage permet de réduire l'amplification, donc d'uti- 
liser une faible valeur de la constante de temps et par là même de décrire à plus grande vitesse la zone spectrale étudiée.

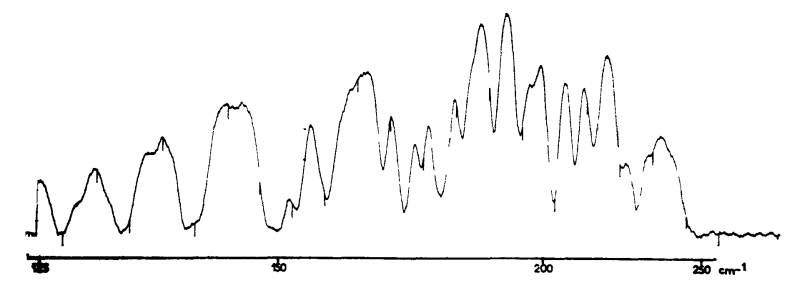

FIG. 15.

Vapeur d'eau atmosphérique : parcours $1 \mathrm{~m}$; $p_{0}=1$ atm.

Réseau : $15 \mathrm{t} / \mathrm{mm} ; f=1 \mathrm{~mm} ; \mathrm{RC}=15^{\circ}$ à $1^{\prime}$ heure ; $38 \mu<\lambda<76 \mu$.

Filtres : $1,25 \mathrm{~mm}$ polythène $+0,5 \mathrm{~mm}$ quartz $+\mathrm{FLi}$.

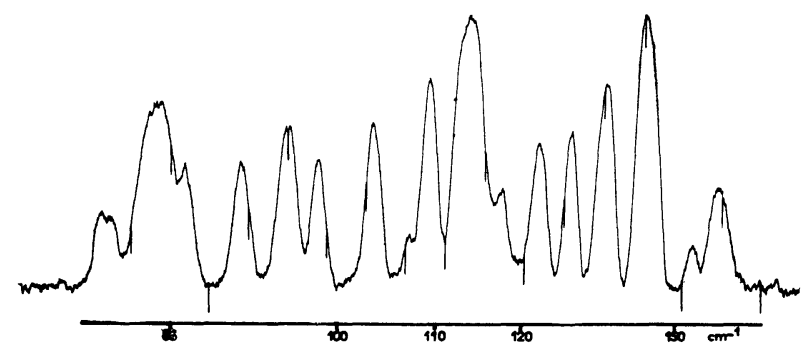

FIG. 16.

Vapeur d'eau atmosphérique; parcours $1 \mathrm{~m}$; $p_{0}=1$ atm.

Réseau : $8 \mathrm{t} / \mathrm{mm} ; f=1 \mathrm{~mm} ; \mathrm{RC}=40 \mathrm{~s} ; v=10^{\circ}$ à l'heure ; $56 \mu<\lambda<112 \mu$.

Filtres: $\mathrm{FLi}+\mathrm{BaF}_{2}$.

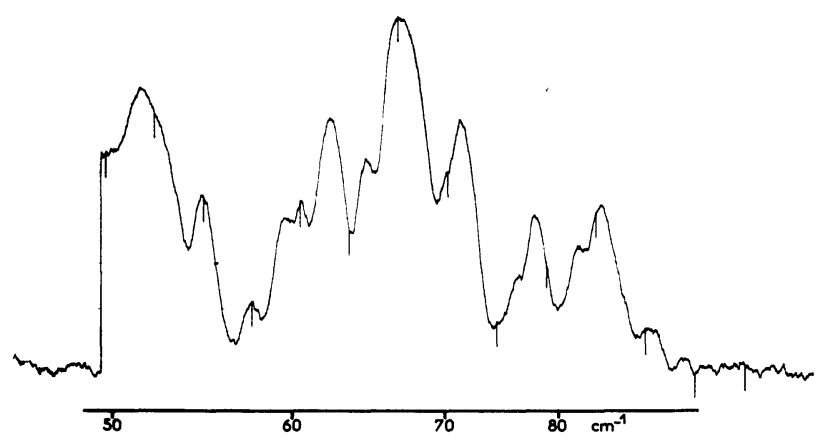

FIG. 17.

Vapeur d'eau atmosphérique; parcours $1 \mathrm{~m}$; $\mathrm{p}_{0}=1 \mathrm{~atm}$.

Réseau : $4 \mathrm{t} / \mathrm{mm} ; f=1 \mathrm{~mm} ; \mathrm{RC}=66 \mathrm{~s} ; v=10^{\circ}$ à l'heure; $98 \mu<\lambda<196 \mu$.

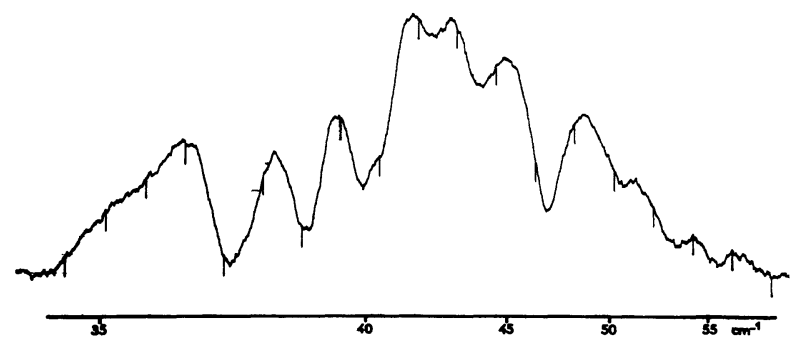

FIG. 18.

Vapeur d'eau atmosphérique; parcours $1 \mathrm{~m}$; $\mathrm{p}_{0}=1$ atm.

Réseau : $4 \mathrm{t} / \mathrm{mm} ; f=2 \mathrm{~mm} ; \mathrm{RC}=100 \mathrm{~s} ; v=70$

à l'heure ; $150 \mu<\lambda<300 \mu$.

Filtres : $\mathrm{FI}_{\mathbf{i}}+\mathrm{BaF}_{2}+\mathrm{KBr}+\mathrm{KCl}+[\mathrm{ICs}]$.

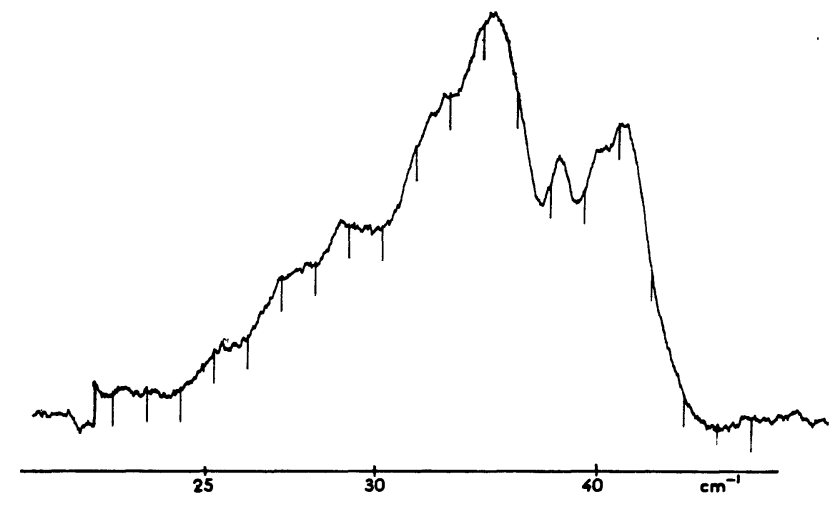

FIG. 19.

Vapeur d'eau atmosphérique; parcours $1 \mathrm{~m}$; $\mathrm{p}_{0}=1$ atm.

Réseau : $2,5 \mathrm{t} / \mathrm{mm} ; f=2 \mathrm{~mm} ; \mathrm{RC}=150 \mathrm{~s}$; $v=7^{\circ}$ à l'heure ; $220 \mu<\lambda<440 \mu$.

Filtres : $\mathrm{FLi}+\mathrm{BaF}_{2}+\mathrm{KCl}+\mathrm{ITl}+[\mathrm{ITl}]$.

Conclusion. - Les filtres décrits, associés selon le tableau I ci-après, nous permettent de couvrir l'intervalle spectral de 40 à 440 microns en laissant des zones de recouvrement suffisantes; les réseaux sont utilisés dans leur premier ordre et au voisinage du blaze. A notre connaissance, c'est la première fois que cette région est couverte par l'utilisation exclusive de ce type de filtres par transmission qui n'étaient jusque-là employés qu'en complément d'un filtrage par réseaux échelettes, réseaux grilles ou réseaux de polythène par transmission. Ce mode de filtrage devrait permettre de réaliser des spectromètres dont l'optique serait simplifiée ou de prolonger vers les basses fréquences des spectromètres déjà réalisés sans modifier le schéma optique. 


\section{TABLEAU I}

\begin{tabular}{|c|c|c|c|c|}
\hline INTER & VALLE & PECTRAL & & SEAU \\
\hline 263 & -131 & $\mathrm{~cm}^{-1}$ & 15 & $\mathrm{t} / \mathrm{mr}$ \\
\hline 172 & -86 & $\mathrm{~cm}^{-1}$ & 8 & $\mathrm{hr}$ \\
\hline 100 & -50 & $\mathrm{~cm}^{-1}$ & 4 & $\mathrm{t} / \mathrm{mr}$ \\
\hline 62 & -31 & $\mathrm{~cm}^{-1}$ & 4 & $\mathrm{t} / \mathrm{mr}$ \\
\hline 45,5 & $5-22,77$ & $\mathrm{~cm}^{-1}$ & 2,5 & $\mathrm{t} / \mathrm{mr}$ \\
\hline
\end{tabular}

FiLtRes

$0,5 \mathrm{~mm}$ de quartz $+1,25 \mathrm{~mm}$ de poudre de polythène pastillée $+\mathrm{FLi}$

(Ces filtres restent à demeure sur le trajet du faisceau.)

$\mathrm{BaF}_{2}$

$\mathrm{BaF}_{2}+\mathrm{KGl}+\mathrm{RbBr}$

$\mathrm{BaF}_{2}+\mathrm{KCl}+\mathrm{RbBr}+\mathrm{RbCI}+[\mathrm{ICs}]$

$\mathrm{BaF}_{2}+\mathrm{KCl}+\mathrm{RbBr}+[\mathrm{ICs}]+[\mathrm{TlI}]$

Les filtres notés [ ] sont obtenus à partir de poudre de l'halogénure alcalin pressée en lamelles très fines, et maintenue entre deux feuilles de polythène.

Manuscrit reçu le $1^{\mathrm{er}}$ juin 1967.

\section{BIBLIOGRAPHIE}

[1] Sournia (A.), D.E.S., Perpignan, 1965.

[2] Delorme (P.), LoRenzeli I (V.), Quintard (P.), C. R. Acad. Sci., Paris, 1965, 261, 3331.

[3] DELORME (P.), LORENZELII (V.), DeNISSELLE (F.), J. Chim. Physique, 1967, 64, 591.

[4] Delorme (P.), Hadni (A.), C. R. Acad. Sci., Paris' 1961, 252, 1299.

[5] Delorme (P.), Thèse, Paris, 1963.

[6] Adams (D. H.), Spectrochim. Acta, 1962, 18, 1039.

[7] KNEUbÜHL (F. K.), MOSER (J. F.), STEFFEN (H.), J. Opt. Soc. Amer., 1966, 56, 6, 760.

[8] LORENZELII (V.), Rev. Opt., 1963, 3, 129.

[9] Makino (I.), IWASAKI (T.), IWAHAShi (I.), Jap. J. Appl. Physics, 1965, 4, Suppl. 1, 369.

[10] M LLER (K. D.), Tomaselili (V. P.), Skube (L. R.), McKenna (B. K.), J. Opt. Soc. Amer., 1965, 55, $10,1233$.

[11] Yaroslavsky (N. G.), Zheludov (B. A.), StaneVICH (A. E.), Opt. $i$ Spektr., 1956, 1, 507.

[12] Yaroslavsky (N. G.), Uspekhi Fiz. Nauk., 1957, 62, 159.

[13] Yoshinaga (H.), Fujita (S.), Minami (S.), MiTSUISHI (A.), OETJEN (R. A.), YAMADA (Y.), J. Opt. Soc. Amer., 1958, 48, 5, 315.

[14] Hadni (A.), Janot (C.), Rev. Opt., 1960, 39, 10, 451.

[15] OetJen (R. A.) et coll., J. Opt. Soc. Amer., 1952, 42, 8, 559 .

[16] BLOOR (D.), DEAN (T. J.), JONES (G. O.), MARTin (D. H.), Mawer (P. A.), Perry (C. H.), Proc. Roy. Soc., London, 1961, A 260, 510.

[17] BLOOR (D.), Spectrochim. Acta, 1965, 21, 595.

[18] Blaine (L. R.), J. Res. Nat. Bur. Std., 1963, 67 C, 3, 207.

[19] McCubbin (T. K.), Sinton (W. M.), J. Opt. Soc. Amer., 1950, 40, 8, 537.

[20] Farmer (C. B.), Key (P. J.), Appl. Optics, 1965, 4, 9, 1051.

[21] Hadni (A.), Decamps (E.), Rev, Univ, Mines, 1959, $9^{\mathrm{e}}$ série, 15,423 .
[22] Hadni (A.), Decamps (E.), C. R. Acad. Sci., Paris, 1959, 249, 2048.

[23] Ginsburgh (N.), Final report A F 19 (604), 2443, Syracuse University, 1961.

[24] Murzin (V. N.), Optics and Spectroscopy, 1962, 13, 6, 467.

[25] Perry (C. H.), Quart. Progress. report, no 70, 15 juillet 1963, Mass. Inst. Technol.

[26] Plyier (E. K.), AcQuista (N.), J. Chem. Phys., 1955, 23, 752 .

[27] Russei, (J. W.), Strauss (H. L.), Appl. Optics, 1965, 4, 9, 1131 .

[28] Zhukov (A. G.), Opt. and Spectroscopy, 1964, 17, 2, 148.

[29] Plylei (E. K.), Yates (D. J. C.), Gebbie (H. A.), J. Opt. Soc. Amer., 1962, 52, 8, 859.

[30] Boldt (W.), Reiman (H.), Ann. Phys., 1960, 6, 293.

[31] McCubbin (T. K.), Sinton (W. M.), J. Opt. Soc. Amer., 1952, 42, 2, 113.

[32] LichtenbeRg (A. J.), SeSNic (S.), J. Opt. Soc. Amer., 1966, 56, 1, 75.

[33] Eber' (H.), Ann. Phys. Chem., 1889, 38, 489.

[34] Czerny (M.), Turner (A. F.), Z. Physik, 1930, 61, 792.

[35] Fastie (W. G.), J. Opt. Soc. Amer., 1952, 42, 641.

[36] Fastie (W. G.), J. Opt. Soc. Amer., 1952, 42, 647.

[37] HadnI (A.), Ann. Phys., 13e série, 1956, 4, 765.

[38] LecomTe (J.), Sel. Sci. Pap. Inst. Sup. di Sanita, $1959,31,3$.

[39] Golay (M. J. E.), Rev. Sci. Inst., 1947, 18, 5, 357.

[40] Golay (M. J. E.), Rev. Sci. Inst., 1949, 20, 11, 816.

[41] Yosihara (K.), Science of light, 1958, 7, 3, 67.

[42] Parodi (M.), Thèse, Paris, 1938.

[43] CZERny (M.) et RöDER (H.), Evgebn. exakt. Naturw., 1938, 17, 70 .

[44] Mitsuishi (A.), Yamada (Y.) et YoshinaGa (H.), J. Opt. Soc. Amer., 1962, 52, 14.

[45] White (J. U.), J. Opt. Soc. Amer., 1947, 37, 713. 
[46] Stamm (R. F.), Whalen (J. J.), J. Opt. Soc. Amer., 1946, 36, 2.

[47] HadnI (A.), Thèse, Paris, 1955.

[48] Janot (C.), Thèse, Nancy, 1963.

[49] Mitsuishi (A.), OTsuka (Y.), Fujita (S.) et YoshiNAGA (H.), Jap. J. of Appl. Physics, 1963, 2, 9, 574.

[50] RenK (K.) et Genzel, (L.), Appl. Optics, 1962, 1, 5, 643.

[51] UlRICH (R.), RENK (K. F.) et GeNZEL (L.), Trans. I.R.E.E., 1963, M.T.T. 11, 363.

[52] Casey (J. P.) et Lewis (E. A.), J. Opt. Soc. Amer., 1952, 42, 971.

[53] MUlieR (R.), Z. Naturforsch., 1953, 8 A, 56.

[54] LARSEN (T.), IRE. MTT, 1962, 10, 191.

[55] Moliek (K. D.) et McKnight (R. V.), J. Opt. Soc. Amer., 1965, 55, 9, 1075.

[56] Yamada (Y.), Mitsuishi (A.), Yoshinaga (H.), J. Opt. Soc. Amer., 1962, 52, 1, 17.

[57] ACKermann (F. W.), Ann. Phys. Lpz., 1940, 37, 442.
[58] Molier (K. D.), McMahon (D. J.) et SMiTh (D. R.), Appl. Optics, 1966, 5, 3, 403.

[59] Delorme (P.) et LoRenzeliLi (V.), J. Phys. Radium, 1962, 23, 589.

[60] LECOMTE (J.), Handbuch der Physik, t. XXVI, Berlin, 1958.

[61] MCCarTy (D. E.), Appl. Optics, 1965, 4, 3, 317.

[62] Green (J. H. S.), Kynaston (W.), Gebbie (H. A.), Spectrochim. Acta, 1963, 19, 807.

[63] SAEki (S.), Bull. Chem. Soc. Jap., 1962, 35, 326, 332.

[64] Jakobsen (R. J.), Brasch (J. W.), J. Amer. Chem. Soc., 1964, 86, 3571.

[65] Hadni (A.), C. R. Acad. Sci., Paris, 1954, 238, 573.

[66] Fraser (R. D.), J. Opt. Soc. Amev., 1953, 43, 10, 929.

[67] Hadni (A.), Decamps (E.), Janot (C.), Communication $8^{\mathrm{e}}$ Coll. int. Bologne, 1959.

[68] Holmyday (P.), J. Sci. instr., 1954, 31, 261.

[69] Guigere (P. A.) et Badger (R. M.), J. Opt. Soc. Amer., 1948, 38, 987.

[70] Gaebler (O. H.), J. Opt. Soc. Amer., 1953, 43, 705. 